\title{
Analytischer "Scharfsinn" und geschulte Intuition im Dialog
}

\author{
Fallverstehen und sozialpädagogische Diagnostik als Schlüsselprozess \\ professioneller Urteilsbildung
}

Im Kontext professioneller Urteilsbildung in der Sozialen Arbeit stellen Fallverstehen und sozialpädagogische Diagnostik einen Schlüsselprozess dar. Fachlicher Anspruch ist es dabei, hochkomplexe Lebens- und Problemlagen fundiert einzuschätzen und darauf aufbauend mit Adressat_innen tragfähige Hilfen zu vereinbaren und zu gestalten. Dieser Prozess vollzieht sich in dem Wissen, dass sozialpädagogische Diagnostik immer einen hypothetischen Charakter hat. Welchen Anforderungen muss in einem solchen Verständnis die professionelle Urteilsbildung genügen, um als fachlich qualifiziert gelten zu können?

$\mathrm{P}$ rofessionelle Urteilsbildung in der Sozialen Arbeit fragt schlicht übersetzt danach, auf welchen Wegen, mit welchen Landkarten und welcher Ausstattung die Profession, d. h. Fachkräfte eingebunden in ihre Organisationen, bezogen auf ihren Gegenstandbereich zu fachlich begründeten Einschätzungen und Positionen kommen, die das weitere Handeln in einem Fall leiten. Wesentlich verbindet sich damit in der fachlichen Debatte die Frage nach Fallverstehen und sozialpädagogischer Diagnostik. Dies wird hier als Schlüsselprozess professioneller Urteilsbildung in einem spezifischen Handlungskontext verstanden, denn ein Problem, das nicht verstanden wird, kann auch nicht „erfolgverspre-

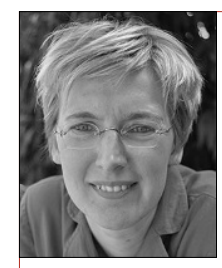

\section{Sabine Ader}

Katholische Hochschule Nordrhein-Westfalen Katholische Fachhochschule gGmbH,

Münster, Deutschland

*1967; Dipl. Päd., Supervisorin (DGSv), Professorin für Theorien und Konzepte Soziale Arbeit, Katholische Hochschule NRW, Abteilung Münster. Langjährig tätig in Beratung, Fortbildung, Supervision und (Praxis-)Forschung.

s.ader@katho-nrw.de
Zusammenfassung Der Beitrag entfaltet Fallverstehen und sozialpädagogische Diagnostik als Schlüsselprozess professioneller Urteilsbildung. Als Ausgangspunkt rücken der besondere Handlungskontext sowie der spezifische, professionelle Blick in der Sozialen Arbeit in den Fokus. Im Anschluss werden die mit der sozialpädagogischen Perspektive verbundenen Kernbestandteile professioneller Urteilsbildung skizziert und ihre Verbindung zueinander aufgezeigt. Abschließend geht es um die fachlichen Anforderungen an dieses anspruchsvolle Vorhaben.

Schlüsselwörter Strukturelle Ungewissheit, Fallverstehen und sozialpädagogische Diagnostik, Wissen, Geschulte Intuition, Dialog, Reflexive Professionalität, Kollegiale Beratung chend" bearbeitet werden. Erste Herausforderung dabei ist der konkrete Handlungskontext.

Strukturelle Ungewissheit als Normalfall der Praxis Fachkräfte der Sozialen Arbeit sind täglich mit der Komplexität und Offenheit von Lebensvollzügen sowie oftmals mit herausfordernden Lebenslagen und Bewältigungsstrategien von Adressat_innen konfrontiert. Ihr Handeln vollzieht sich in der strukturellen Unsicherheit sozialer Situationen, die einzigartig sind und immer wieder neu zunächst verstanden und dann bearbeitet werden müssen. Unvorhersehbarkeit, Widersprüchlichkeit und Kontingenz sind für solche Handlungsvollzüge charakteristisch (Dewe und Otto 2018b). Diese anspruchsvolle Ausgangssituation und die damit einhergehende Notwendigkeit von Bewerten und Handeln in uneindeutigen Kontexten, zudem häufig unter zeitlichen Druck, gilt nahezu für alle Bereiche der Sozialen Arbeit. Nachfolgende Ausführungen fokussieren jedoch im Besonderen auf die Einzelfallarbeit sowie exemplarisch auf die Kinder- und Jugendhilfe, in der der Diskurs über Fallverstehen und sozialpädagogische Diagnostik intensiv geführt wird.

„Fälle“ (i.S. erkannter Hilfebedarfe) zu bearbeiten ist die wesentliche Aufgabe und Tätigkeit derjenigen Fachkräfte in der Kinder- und Jugendhilfe, die vor allem mit den sogenannten Hilfen zur Erziehung und Fragen des Kinderschutzes befasst sind. Fälle sind ein komplexes und kompliziertes Bedingungsgefüge: einerseits geprägt durch eine akute Problemlage, in der seitens der Fachkräfte ebenso Anliegen und Anfragen aufzunehmen sind, wie Zuständigkeiten zu klären und Zugänge zu finden. Andererseits verweisen schon erste Gespräche, Informationen und Eindrücke auf Vorgeschichten und 


\section{Extrablick: Urteilsbildung in der Sozialen Arbeit}

Hintergründe - sowohl in den Lebensgeschichten von Kindern oder Jugendlichen, aber auch von ihren Eltern oder zentralen Bezugspersonen. Und sie verweisen auf unterschiedliche Erfahrungen von Adressat_innen mit dem Hilfesystem, die als hilfreich oder/und leidvoll erlebt wurden.

Fallbearbeitung in der Kinder- und Jugendhilfe zeichnet sich in dem Kontext dadurch aus, dass in einem dialogischen Prozess mit allen Beteiligten sowohl konkret als auch grundlegend Entscheidungen getroffen und begründet werden müssen, die meist tief in das Leben der Adressat_innen hineinreichen. Es geht um fundierte Einschätzungen zu Leistungsansprüchen, zur konkreten Gestaltung von Unterstützung, aber auch zu Eingriffen in elterliche Rechte, wenn dies zum Schutz ihrer Kinder erforderlich erscheint. Derartige Entscheidungsprozesse gehen weit über den Augenblick hinaus und sind folgenreich für Entwicklungschancen und Lebensperspektiven der betroffenen jungen Menschen.

Für die entscheidungsorientierte Bearbeitung hochkomplexer Fallkonstellationen verantwortlich zu sein, stellt eine enorm hohe Anforderung und Anstrengung für Fachkräfte dar, zumal sich dies in dem Wissen vollzieht, dass eigene Entscheidungen lediglich durch prognostische Einschätzungen „gesichert" werden können und damit immer einem Ungewissheitshorizont unterliegen. Der berechtigte und individuell verständliche Wunsch nach Eindeutigkeit für die eigenen Arbeitszusammenhänge kann nicht im Sinne „objektiv richtiger Entscheidungen aufgrund einer eindeutigen Faktenlage“ eingelöst werden. Die professionelle Hilfegestaltung und damit verbunden die Balancierung der Vielfalt menschlicher Lebensvollzüge und oft widerstreitender Interessen ist somit eine wesentliche Kompetenz und Ausweis enorm anspruchsvoller Fachlichkeit in der Sozialen Arbeit. Schlüsselprozesse für das Handeln sind dabei das Wahrnehmen, Verstehen und Bewerten von Belastungs- und Krisensituationen - also die professionelle Urteilsbildung.

\section{Fall-Verstehen und sozialpädagogische Diagnostik als Schlüsselkompetenz}

Skizziert wurde, dass soziale Situationen immer gekennzeichnet sind durch mehrdeutige Verhältnisse und subjektiv jeweils berechtigte Wirklichkeitsdeutungen. Es gibt

- keine eindeutige Zuordnung von Ursachen und Wirkungen

- und von Problemen und Lösungen,

- eine immer nur begrenzte Aussagefähigkeit einmal gewonnener Erkenntnisse für zukünftige Entscheidungen
- und einen ebenso begrenzten professionellen Einfluss auf das Ergebnis, d. h. die Wirkung von professionellen Entscheidungen und Handlungen (Ader und Schrapper 2020).

Oftmals gibt es somit eine Menge Wahrscheinlichkeiten, aber wenige Eindeutigkeiten; zudem immer Facetten sozialer Situationen, die individuell nicht erkannt werden können.

Generell wird menschliches Verhalten geleitet durch subjektiv geprägte Wahrnehmungen, damit verbundene Bewertungen und insbesondere Emotionen, die das Denken und Handeln maßgeblich und i. d. R. unbemerkt formieren (Gigerenzer 2008; Ciompi 1997). Dies ermöglicht Menschen durch entwickelte Routinen, die vielfältigen Anforderungen des Alltags zu bewältigen. Prozesse des Nachdenkens werden abgekürzt. Gleichzeitig aber verengen solche entlastenden Abkürzungen den Blick und klammern Deutungen aus, die jenseits der eigenen Wahrnehmungsroutinen liegen. Die individuelle Sichtweise wird schnell für die einzig „richtige“ gehalten.

Professionelles Verstehen sowie wissenschaftlicher Erkenntnisgewinn müssen sich von dieser alltäglichen Praxis unterscheiden. Es gilt, Wahrnehmungen und Erkenntnisse auf bewussten Wegen und für Dritte nachvollziehbar zu gewinnen. Dabei ist die Vielfalt von Perspektiven auf eine Situation systematisch zu entfalten, um sukzessiv Hinweise darauf zu erhalten, wie etwas fachlich eingeschätzt werden kann und ob diese Bewertung (verstanden als fundiert begründete Hypothesen) einer intersubjektiven Überprüfung standhält. Die methodisch zu lösende Grundoperation ist der Umgang mit Komplexität. Wie jeder Prozess empirischer Erkenntnisgewinnung haben auch Prozesse des professionellen Verstehens und Beurteilens ein erkenntnislogisches Grundproblem zu lösen (vgl. Dewe und Otto 2018a):

- Zuerst muss der „analytische Blick“ erweitert, die Komplexität erhöht werden, damit überhaupt etwas Neues wahrgenommen werden kann und nicht nur schon Bekanntes bestätigt wird.

- Ist auf diese Weise ausreichend Material für erweiternde Erkenntnisse gewonnen, muss der Blick wieder enggeführt werden, um aus der Vielfalt der Wahrnehmungen die für zentral gehaltenen Zusammenhänge herauszuarbeiten. Gelingt diese Reduktion von Komplexität nicht, so verschwinden mögliche Befunde in einer Vielzahl unverbundener und unverstandener Beobachtungen. (Abb. 1).

In diesem Zusammenhang ist die fallanalytische Arbeit einerseits eine Verstehensleistung und erkenntnistheo- 


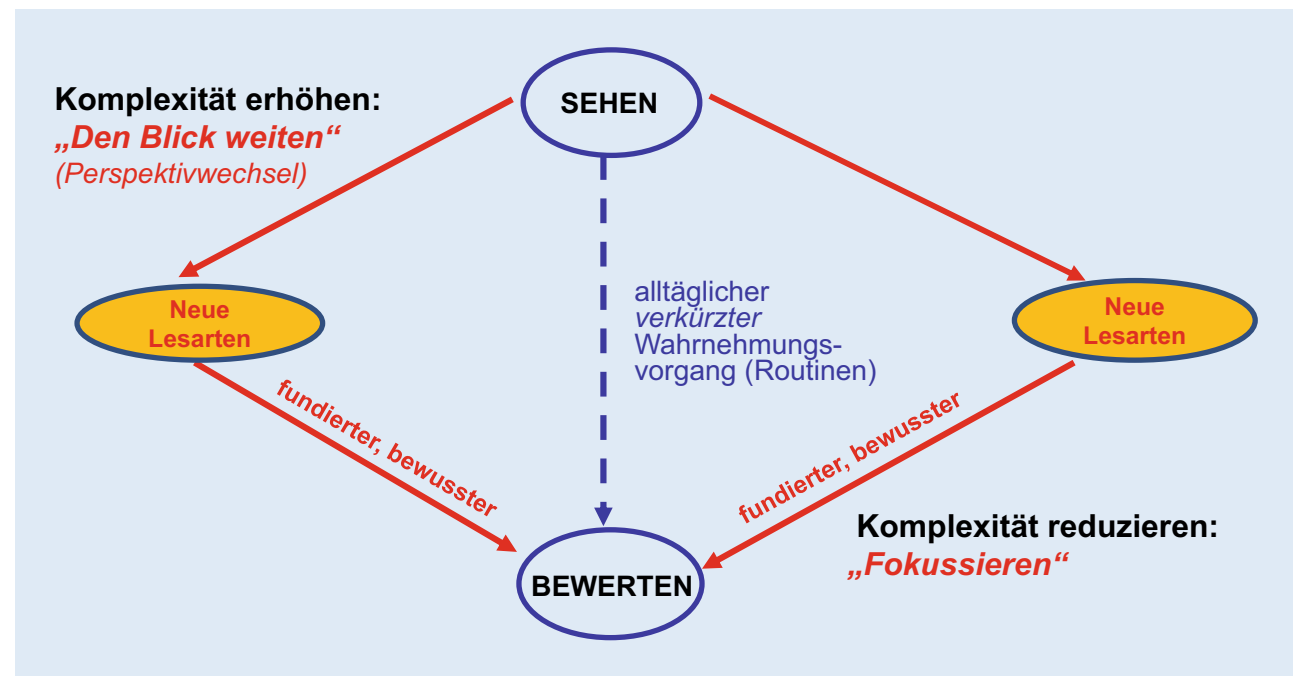

Abb. 1 Grundbewegung professionellen Erkenntnisgewinns (eigene Abbildung)

retisch einzuordnen in die lange Tradition der (geisteswissenschaftlichen) Hermeneutik (vgl. z. B. Mollenhauer und Uhlendorff 1992; Oevermann 2000). Mit dem Konzept der „stellvertretenden Deutung“ (ebd.) und der Figur der „immanenten Kunstlehre des Fallverstehens" (Gildemeister 1992) wurden zentrale Orientierungspunkte für die Entwicklung einer professionellen Deutungskompetenz theoretisch markiert. Die Gleichzeitigkeit von Theorieverstehen und Fallverstehen ist nach Gildemeister dabei grundlegend, wobei die Kunstlehre des Fallverstehens in einem langdauernden Prozess professioneller Identitätsbildung eingeübt werden muss (vgl. ebd. 1992) und sich in der Berufspraxis Sozialer Arbeit idealerweise in einem entsprechenden professionellen Habitus ausdrückt (vgl. auch Becker-Lenz et al. 2012). In dieser Linie hermeneutischer Erkenntnisprozesse stehend, bedarf es für das konkrete Verstehen einer wissensbasierten, reflexiv geschulten wie auch mitmenschlichen Anstrengung, einfühlend nachzuvollziehen, wie sich Not und Bedrängnis für Menschen anfühlen und welche inneren Logiken sie antreiben. Geleitet wird ein (sozial)pädagogisches Fallverstehen und Diagnostizieren folglich vor allem von der Frage nach dem Sinn und der Funktion, die ein als auffällig oder dysfunktional wahrgenommenes Verhalten in der Lebenspraxis, der Bildungsgeschichte und den (Über-)Lebensstrategien von (jungen) Menschen hat. Der Eigensinn und die Widersprüche, die Spannungen und Brüche in der Lebens- und Lerngeschichte eines Menschen in ihrem subjektiven Sinn zu entschlüsseln, ist der entscheidende Zugang eines explizit sozialpädagogischen Verstehens und Diagnostizierens - anders also als z. B. die Aufgabe psychiatrischer Diagnostik. Und nicht selten muss hier auch das stellvertretend in Sprache gefasst und dem Dialog zugänglich gemacht werden, was Menschen selbst bislang unzugänglich oder unsagbar war.
Zum anderen bedarf es als Gegenüber und Ergänzung der analytischen und „scharfsichtigen“ Anstrengung, die Dinge „beim Namen zu nennen“. Hier geht es vor allem um die Zuordnung zu einem anerkannten Allgemeinen (Müller 2012), für das unterschiedliche Wissenstypen und Wissensformen sowie geklärte theoretische Begriffe ebenso unverzichtbar sind wie die Fähigkeit, in Distanz zum Fallgeschehen zu eigenständigen Positionierungen zu kommen. Neben der Verstehensleistung müssen begründete Hypothesen von Fachkräften erarbeitetet werden, die Situationen und Sachverhalte möglichst objektiv (d. h. kriteriengeleitet) und nachvollziehbar bewerten. Systematische, wissensbasierte Beobachtungs- und Dokumentationsinstrumente als eine Quelle der kollegialen Beratung gehören hier durchaus dazu.

Für Fallverstehen und sozialpädagogische Diagnostik bedarf es damit sowohl einer analytisch-einordnenden Kompetenz als auch einer verstehenden und kommunikativen Kompetenz, da eine möglichst sensible und vielschichtige professionelle Wahrnehmung und Deutung an die „Qualität der Begegnung der Subjekte in einer Szene gebunden“ ist (Heiner 2004, S. 93), also auch ein Ergebnis von Einfühlung und emotionaler Resonanz. Letztlich ist fallanalytische Arbeit und damit der Prozess professioneller Urteilsbildung immer auch ein Beziehungsgeschehen zwischen den Adressat_innen und den fallbeteiligten Fachkräften - zwar im institutionellen Kontext und dennoch immer affektiv aufgeladen und davon geprägt.

\section{Die Basis für Verstehen und Beurteilen:} Wissen und geschulte Intuition im Dialog Begreift man den Prozess professioneller Urteilsbildung auch als interaktives Beziehungsgeschehen, so sind Fachkräfte und ihre Institutionen Teil des Falls, sobald 


\section{Hintergrund: Verständnis reflexiver Professionalität}

\section{Fallverstehen und}

\section{U sozialpädagogische Diagnostik (U)}

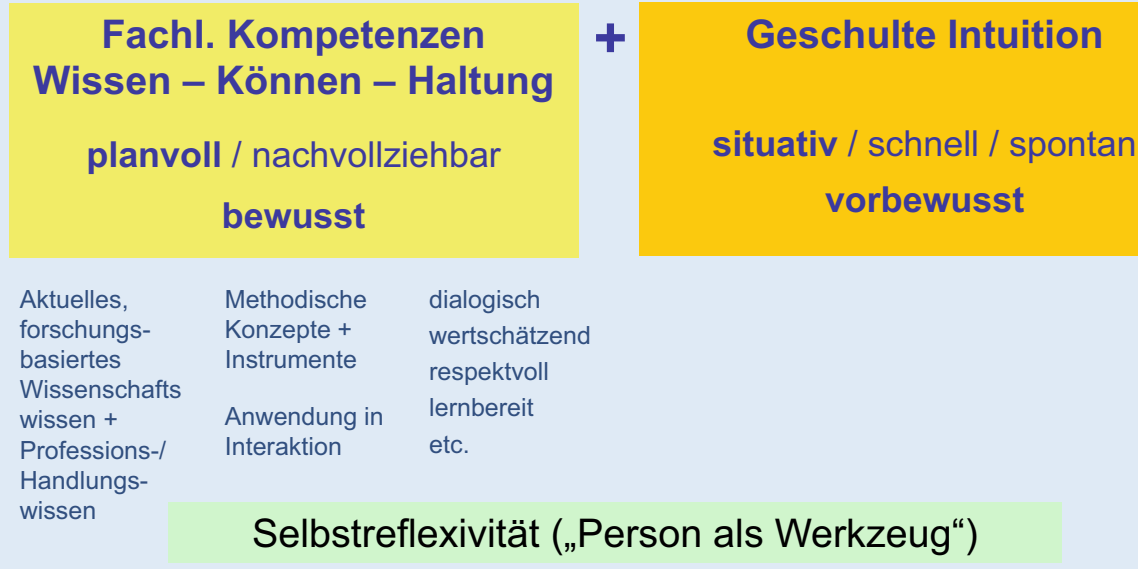

Abb. 2 Grundkompetenzen fachlicher Bewertung (eigene Abbildung) eine schwierige Lebenssituation zum Fall wird. Der damit verbundene Fallbegriff (vgl. Ader 2006) und daraus folgend der Gegenstand für Verstehen und Beurteilen zieht ein spezifisches Konzept von Fallverstehen und sozialpädagogischer Diagnostik nach sich, das in diesem Beitrag nicht weiter entfaltet werden kann (vgl. ausführlich Ader und Schrapper 2020).

Konzeptionell und methodisch - so viel sei dazu benannt - werden darin neben dem Blick auf das System der Adressat_innen die institutionelle Eingebundenheit Sozialer Arbeit sowie die Beziehungsdimension sozialpädagogischen Handelns bewusst in den Erkenntnisprozess einbezogen. Skizziert werden soll hier allerdings die fachliche Basis dafür: Welche Grundkompetenzen für Prozesse fachlicher Bewertung werden gebraucht und welche Anforderungen stellt dies an die Praxis Sozialer Arbeit? (Abb. 2).

Professionelle Beurteilungsprozesse sind aufgrund ihrer Fehleranfälligkeit und ihres ungewissen Ausgangs ein prinzipiell riskantes Vorhaben. Die „Nichtstandardisierbarkeit gilt als genuiner Kern, aber auch als das große Problem der Professionellen“ (Bastian 2019, S. 30). Aufgrund der Überkomplexität lebensweltlicher Situationen kann zur Verfügung stehendes fachliches Wissen nicht regelhaft verwendet werden. Stattdessen geht es um einen kompetenten und mindestens im Nachgang erklärbaren Umgang mit „diffusen Situationen“ und „begrenzten Durchschaubarkeiten“ (vgl. Klomann et al. 2019, S. 5). Aufgrund dessen ist für die Fallarbeit ein Professionsverständnis Reflexiver Sozialpädagogik grundlegend, dass die Reflexion und die Fähigkeit zur diskursiven Deutung auf der Basis unterschiedlicher Wissensbestände zum wesentlichen Bezugspunkt professioneller Handlungen macht (vgl. Dewe und Otto 2012). Auf den Schlüsselprozess von Verstehen und Diagnostizieren im Rahmen professioneller Urteilsbildung bezogen, zieht dies für Fachkräfte drei Grundkompetenzen nach sich, die zentral sind und Prozesse professioneller Habitualisierung bedürfen - nicht zuletzt in der akademischen Ausbildung. Es geht um das souveräne Verfügen über unterschiedliche Wissensbestände, eine geschulte Intuition sowie eine hohe Dialogbereitschaft (verknüpft mit der Fähigkeit zu dialektischem Denken). Erstens ist professionelles Wissen die Basis für die reflexive Verarbeitung ungewisser Handlungskontexte und Ambivalenzen. Ein solches, fortwährend aktuell zu haltendes Wissen in der Sozialen Arbeit besteht aus unterschiedlichen Wissensbeständen, die begründbar einzusetzen sind. Dabei geht es einerseits um systematisches Wissenschaftswissen, das einem Begründungszwang unterliegt und nicht unmittelbar auf konkrete Handlungssituationen übertragen werden kann, sowie in Ergänzung dazu um praktisches Handlungswissen (vgl. Dewe und Otto 2012). Nach Dewe/Otto ist für professionalisiertes Handeln nicht allein die wissensbasierte Kompetenz konstitutiv. Neben regelhaftem Wissen („Allgemeinverstehen“) braucht es die situativ aufzubringende reflexive Fähigkeit konkrete lebensweltliche Problemsituationen wissensbasiert zu durchschauen und zu erklären. Die gewonnenen Einsichten zu einer 
immer einmaligen Situation („Fallverstehen“) sind zudem den Adressat_innen im Verlauf gemeinsamer Verständigung zur Verfügung zu stellen als Basis für von ihnen selbst zu verantwortende Entscheidungen. Professionswissen bedarf also beider Bestandteile professionellen Wissens und erfordert deren Relationierung im Kontext des konkreten Handlungsvollzugs. Zu nutzen ist das Wissen dabei - metaphorisch beschrieben - als Landkarte, nicht als Werkzeugkoffer.

Neben der Anwendung bewussten Wissens ist zweitens die geschulte Intuition eine - als entwickelte professionelle Kompetenz bislang unterbewertete - Form des Erkenntnisgewinns, die vor- und/oder unbewusst verläuft und über eine schnelle Reduktion von Komplexität eine Situation ganzheitlich erfasst. Diese Erkenntnisgewinnung stellt eine Art situativer Diagnostik dar und speist sich aus unterschiedlichen Quellen. Sie greift auf verschiedene Wissensbestände zurück, die ein Mensch in sich trägt $u n d$ aktuell in einer Situation erlebt: (Lebens-) Erfahrungen und Erlebnisse, angeeignetes (Fach-)Wissen, situative Emotionen und Resonanzen auf das Gegenüber scheinen die wesentlichen Bauteile (Gigerenzer 2008).

Generell zeigt sich menschliche Intuition ad hoc in einer konkreten Situation und ist nicht herstellbar. Häufig ist sie mit einem (zügigen) Handlungsimpuls gekoppelt. Sie ist nicht an fachliches Wissen gebunden, sondern stärker an die Summe individueller Erfahrungen und Erlebnisse, auf die un-/vorbewusst zurückgegriffen wird. Und sie ist keineswegs auf psychosoziale Situationen beschränkt.

Ist die menschliche Intuition nicht geschult, d. h. durch selbsterfahrungsbezogenes Lernen qualifiziert, so kann sie in professionellen Handlungskontexten Sozialer Arbeit riskant sein, weil sie dann auch auf eigene unverstandene Lebenserfahrungen und -themen zurückgreift. Geschulte Intuition zu elaborieren bedeutet, im Laufe der beruflichen Entwicklung zunehmend mehr über das eigene Selbst „verfügen“ zu können, d. h. unbewusste Denk- und Handlungsmotive durch kontinuierliche Selbstreflexion und Introspektion (Mertens 2014) nach und nach mehr zu erkennen und sich den damit verbundenen Lernprozessen zu stellen. Dieser (Selbst-)Bildungsprozess ermöglicht den Aufbau von Faustregeln und Heuristiken als Quelle der Intuition, die die dahinterliegenden persönlichen Erfahrungen reflektiert haben bzw. immer wieder neu reflektieren und damit das Risiko reduzieren, dass eigene unverstandene Lebensthemen und Konflikte die wesentliche Quelle für die eigene Intuition sind.

Intuition als fachliche Ressource und Grundkompetenz im Sinne geschulter Intuition kann somit entstehen, wenn die grundlegend menschliche Fähigkeit zur
Intuition in Qualifizierungsprozessen bewusst ausgebildet wird durch die (selbstbezügliche) Reflexion von Entscheidungen und Handlungen, die intuitiv getroffen bzw. umgesetzt wurden. Sie speist sich dann aus unterschiedlichen Quellen. Dazu gehören:

- Differenzierte Wahrnehmungsfähigkeit und hohe Aufmerksamkeit

- Reflektierte (Lebens- und Selbst-) Erfahrung

- Emotionale Präsenz in der Situation

- Fachliche Kompetenz (in den Dimensionen Wissen, Können, Haltung)

- Zudem erscheint es sinnvoll, über eine gewisse Entschlossenheit im Handeln zu verfügen.

All diese Dimensionen müssen qualifiziert werden, wenn die generell menschliche Fähigkeit zur Intuition als ein verantwortlicher Zugang zum professionellen Verstehen sozialer Situationen dienlich sein soll (vgl. z. B. Edding 2011, Stemmer-Lück 2014). Im Kontext Sozialer Arbeit spielen hier Supervision, Kollegiale Beratung, kasuistisches Lernen und nicht zuletzt selbsterfahrungsbezogene (akademische) Ausbildung und Qualifizierung eine wichtige Rolle.

Eine hohe Fähigkeit und Bereitschaft zum Dialog ist die dritte Kompetenz, die für die Fallarbeit und damit verbundene Einschätzungsprozesse eine wesentliche Voraussetzung für das Gelingen ist. „Verstehen geht über Verständigung “ (Thiersch 2014). Der ernsthafte Dialog ist zu suchen und zu führen

- mit den Adressat_innen und dem Ziel aktiver Beteiligung sowie gemeinsamer Verständigung,

- zwischen den Erkenntnisebenen des Wissens und der geschulten Intuition, als gleichsam erforderlichen Kompetenzen für Verstehen und Bewerten (und damit der Auflösung des polaren Denkens),

- mit Kolleg_innen im Rahmen institutionell abgesicherter und methodisch systematisierter Kollegialer Beratung (z. B. Ader und Schrapper 2020). Hier gilt es den fachlichen Anspruch des Zusammenwirkens mehrerer Fachkräfte einzulösen, um ein Gegenüber und Korrektiv für die immer eingeschränkte Wahrnehmungsfähigkeit einzelner zu „sichern“ sowie die fallverantwortlichen Fachkräfte durch institutionalisierte Reflexionsorte zu stärken und zu schützen.

Dialog ist für die genannten Handlungsebenen in der Tradition Bubers (1973) zu verstehen. Fachliches Nachdenken und Handeln ist in diesem Verständnis nicht an einem „Entweder - Oder“ ausgerichtet, sondern an einem „Sowohl - Als auch“. Es geht darum, widerstreitende Kräfte in den Blick zu nehmen, im Diskurs 


\section{Extrablick: Urteilsbildung in der Sozialen Arbeit}

auszutarieren und eine Verständigung zu erzielen, die Widersprüchliches aufnimmt.

\section{Fazit}

Im Kontext professioneller Urteilsbildung muss die Profession sich zukünftig stärker dadurch auszeichnen, dass sie auf nachvollziehbaren Wegen und mit fachlichen fundierten Methoden zu begründeten Bewertungen kommt. Ebenso will und muss sich eine Profession vor allem durch eine exklusive Expertise auszeichnen nicht jede_r sollte können, was die Professionellen vermögen. Dieses professionelle Vermögen spitzt sich zu in der Urteilsfähigkeit, die Optionen und Chancen, vor allem aber die Grenzen und Gefahren des eigenen professionellen Handelns zuverlässig und glaubwürdig einschätzen zu können. Um diesen Nachweis fundierter Urteilsfähigkeit begründet führen zu können, muss die Soziale Arbeit respektive die Kinder- und Jugendhilfe über klare und beschreibbare Konzepte für diese Aufgaben verfügen, die nach Innen und Außen erkennbar und explizierbar sind.

Neben der selbstbewussten Nutzung des eigenen Professionswissens kommt hierbei auch der organisationalen Einbindung und Absicherung des institutionellen Rahmens für diese fachlich hoch anspruchsvolle Arbeit eine besondere Bedeutung zu. Sowohl die Profession als auch die akademische Ausbildung sind gefordert, dies zu fördern und so zur Weiterentwicklung der Profession beizutragen.

\section{Eingegangen. 27. April 2021}

Angenommen. 14. Mai 2021

Funding. Open Access funding enabled and organized by Projekt DEAL.

Open Access. Dieser Artikel wird unter der Creative Commons Namensnennung 4.0 International Lizenz veröffentlicht, welche die Nutzung, Vervielfältigung, Bearbeitung, Verbreitung und Wiedergabe in jeglichem Medium und Format erlaubt, sofern Sie den/die ursprünglichen Autor(en) und die Quelle ordnungsgemäß nennen, einen Link zur Creative Commons Lizenz beifügen und angeben, ob Änderungen vorgenommen wurden.

Die in diesem Artikel enthaltenen Bilder und sonstiges Drittmaterial unterliegen ebenfalls der genannten Creative Commons Lizenz, sofern sich aus der Abbildungslegende nichts anderes ergibt. Sofern das betreffende Material nicht unter der genannten Creative Commons Lizenz steht und die betreffende Handlung nicht nach gesetzlichen Vorschriften erlaubt ist, ist für die oben aufgeführten Weiterverwendungen des Materials die Einwilligung des jeweiligen Rechteinhabers einzuholen.

Weitere Details zur Lizenz entnehmen Sie bitte der Lizenzinformation auf http://creativecommons.org/licenses/by/4.0/deed.de.

\section{Literatur}

Ader, S. (2006). Was leitet den Blick? Wabrnehmung, Deutung und Intervention in der Kinder- und Jugendhilfe. Weinheim, München: Juventa.

Ader, S., \& Schrapper, C. (Hrsg.). (2020). Sozialpädagogische Diagnostik und Fallverstehen in der Jugendhilfe. München: Reinhardt/UTB.

Bastian, P. (2019). Sozialpädagogische Entscheidungen. Professionelle Urteilsbildung in der Sozialen Arbeit. München: Reinhardt/UTB.

Becker-Lenz, R., Busse, S., Ehlert, G., \& Müller-Hermann, S. (Hrsg.). (2012). Professionalität Sozialer Arbeit und Hochschule. Wiesbaden: Springer.

Buber, M. (1973). Das dialogische Prinzip. Gütersloh: Gütersloher Verlagshaus.

Ciompi, L. (1997). Die emotionalen Grundlagen des Denkens. Entwurf einer fraktalen Affektlogik. Göttingen: Vandenhoeck \& Ruprecht.

Dewe, B., \& Otto, H.-U. (2012). Reflexive Sozialpädagogik. In W. Thole (Hrsg.), Grundriss Soziale Arbeit (S. 197-217). Wiesbaden: VS.

Dewe, B., \& Otto, H.-U. (2018a). Wissenschaftstheorie. In H.-U. Otto, H. Thiersch, R. Treptow \& H. Ziegler (Hrsg.), Handbuch Soziale Arbeit. Grundlagen der Sozialarbeit und Sozialpädagogik (6. Aufl. S. 1833-1846). München: Reinhardt.

Dewe, B., \& Otto, H.-U. (2018b). Profession. In H.-U. Otto, H. Thiersch, R. Treptow \& H. Ziegler (Hrsg.), Handbuch Soziale Arbeit. Grundlagen der Sozialarbeit und Sozialpädagogik (6. Aufl. S. 1191-1197). München: Reinhardt.

Edding, C. (2011). Auch die Einfühlung will verstanden sein. Empathie verstehen. Zeitschrift Supervision, 1/2011, 13-22.

Gigerenzer, G. (2008). Bauchentscheidungen. Die Intelligenz des Unbewussten und die Macht der Intuition. München: Bertelsmann.

Gildemeister, R. (1992). Neuere Aspekte der Professionalisierungsdebatte. neue praxis, 3(3), 207-219.

Heiner, M. (2004). Fallverstehen, Typen der Falldarstellung und kasuistische Kompetenz. In R. Hörster, E.-U. Küster \& S. Wolff (Hrsg.), Orte der Verständigung: Beiträge zum sozialpädagogischen Argumentieren (S. 91108). Freiburg: Lambertus.

Klomann, V., Schermaier-Stöckl, B., Breuer-Nyhsen, J., \& Grün, A. (2019). Professionelle Einschätzungsprozesse im Kinderschutz. Forschungsbericht. https://kidoks.bsz-bw.de/frontdoor/deliver/index/docId/1409/file/Einschaetzungsprozesse_im_Kinderschutz_Bericht.pdf

Mertens, W. (Hrsg.). (2014). Handbuch psychoanalytischer Grundbegriffe. Stuttgart: Kohlhammer.

Mollenhauer, K., \& Uhlendorff, U. (1992). Sozialpädagogische Diagnosen I. Über Jugendliche in schwierigen Lebenslagen. Weinheim, München: Juventa.

Müller, B. (2012). Sozialpädagogisches Können. Ein Lehrbuch zur multiperspektivischen Fallarbeit (7. Aufl.). Freiburg: Lambertus.

Oevermann, U. (2000). Die Methode der Fallrekonstruktion in der Grundlagenforschung sowie der klinischen und pädagogischen Praxis. In K. Kraimer (Hrsg.), Die Fallrekonstruktion. Sinnverstehen in der sozialwissenschaftlichen Forschung (S. 58-155). Frankfurt/Main: Suhrkamp.

Otto, H.-U., Thiersch, H., Treptow, R., \& Ziegler, H. (Hrsg.). (2018). Handbuch Soziale Arbeit. Grundlagen der Sozialarbeit und Sozialpädagogik (6. Aufl.). München: Reinhardt.

Stemmer-Lück, M. (2014). Resonanzräume in psychoanalytischen Konzepten. In Fortbildungsinstitut für Supervision - Newsletter, Ausgabe Nov. 2014. Wiesbaden. https://fis-supervision.de/2014/11/07/5-newsletter-desfis/. Zugegriffen: 11. März 2019.

Thiersch, H. (2014). Lebensweltorientierte Soziale Arbeit. Aufgaben der Praxis im sozialen Wandel (9. Aufl.). Weinheim, München: Juventa. 